THE ASTROPHYSICAL JOURNAL SUPPLEMENT SERIES, 92:469-472, 1994 June

(c) 1994. The American Astronomical Society. All rights reserved. Printed in U.S.A.

\title{
DISCOVERY AND OBSERVATIONS BY WATCH OF THE X-RAY TRANSIENT GRS 1915+105
}

\author{
Alberto J. CASTro-Tirado, ${ }^{1,2}$ Søren Brandt, ${ }^{1}$ and Niels Lund \\ Danish Space Research Institute, Gl. Lundtoftevej 7, DK-2800 Denmark \\ IGOR LAPSHOV AND RASHID A. SUNYAEV \\ Space Research Institute, Russian Academy of Sciences, Profsouznaya 84/32, 117296 Moscow, Russia \\ AND
}

Aleksei A. Shlyapnikov, Sergei Guziy, and Elena P. Pavlenko

Crimean Astrophysical Observatory, p/o Nauchny, 19-17, 334413 Crimea, Ukraine

Received 1993 May 4; accepted 1993 December 29

\begin{abstract}
The X-ray transient GRS $1915+105$ was discovered on 1992 August 15 by the WATCH all-sky X-ray monitor on GRANAT. The source is located in the Galactic plane. The initial $\mathrm{X}$-ray light curve is presented, as well as the results of a search for the optical counterpart of the source, which apparently is fainter than 21 mag. Assuming that the source is a low-mass X-ray binary, a lower limit of $1.5 \mathrm{kpc}$ is derived for the distance to the source. The possibility of the source lying inside one of the two molecular cloud complexes along the line of sight is discussed.
\end{abstract}

Subject headings: stars: individual (GRS 1915+105) - X-rays: bursts

\section{INTRODUCTION}

$\mathrm{X}$-ray transients belong to a subgroup of the low-mass X-ray binaries (LMXBs), that are systems formed by a low-mass companion and a compact object which can be a weakly magnetized neutron star or a black hole. Here we report the discovery on 1992 August 15 and follow-up observations of the new transient X-ray source GRS $1915+105$ by the WATCH all-sky monitor on-board GRANAT. The BATSE experiment on the Compton Gamma-Ray Observatory had been observing activity in this region since 1992 May up to energies of $120 \mathrm{keV}$, but without determining the position (Harmon, Paciesas, \& Fishman 1992). Examination of the data from Vela 5B from 1969 May to 1979 June revealed no X-ray flux greater than $0.1 \mathrm{crab}$ lasting longer than 2.3 days (Lochner \& Whitlock 1992). The SIGMA Gamma-Ray Telescope on GRANAT observed the source on 1992 September 23-24 (Finoguenov et al. 1994). The object has been monitored by the WATCH experiment on the ESA EURECA satellite until the end of 1992. At that time, GRS $1915+105$ was still among the brightest sources in the hard X-ray sky (Brandt, Castro-Tirado, \& Lund 1992). Only data from WATCH/GRANAT are included in this paper.

\section{INSTRUMENTATION}

The WATCH experiment (Lund 1985) is an all-sky X-ray monitor on-board the GRANAT satellite, launched in 1989 December 1. It consist of four identical units. Each instrument has a circular field of view of 4 steradians. The instruments are mounted in a tetrahedral configuration covering the whole sky. Position sensitivity is achieved using the rotation collimator principle, with the collimator grids rotating with a frequency of $1 \mathrm{~Hz}$. Each instrument has an effective area of $\sim 30$

\footnotetext{
${ }^{1}$ Copenhagen University Observatory, Øster Voldgade, DK-1350 Copenhagen K, Denmark.

${ }^{2}$ Laboratorio de Astrofisica Espacial y Fisica Fundamental, P.O. box 50727, 28080 Madrid, Spain.
}

$\mathrm{cm}^{2}$ (averaged over the field of view), with the phoswich detectors consisting of interleaved strips of NaI and CsI crystals. The total energy range is approximately $6-150 \mathrm{keV}$.

\section{OBSERVATIONS}

The WATCH data covering the period starting 1992 August 15.5 revealed a new bright $\mathrm{X}$-ray transient in the constellation Aquila, with a flux of $0.35 \mathrm{crab}$ in the $6-15 \mathrm{keV}$ energy band (Castro-Tirado, Brandt, \& Lund 1992). The initial position was improved when a large number of observations were collected. The improved position $\alpha_{1950.0}=19^{\mathrm{h}} 12^{\mathrm{m}} 50^{\mathrm{s}}, \delta_{1950.0}=$ $+10^{\circ} 35^{\prime} 00^{\prime \prime}$ (with 0.5 radius error box at $99 \%$ confidence level) located the source in the Galactic plane, at $1^{\mathrm{II}}=45.1$, $\mathrm{b}^{\mathrm{Il}}=-0.35$. The SIGMA gamma-ray telescope on GRANAT observed the source on 1992 September 23-24. GRS $1915+105$ was found to be $16^{\prime}$ (the center of the SIGMA error box, with a radius of 3.5 at $90 \%$ confidence limit) off from the center of the WATCH error box (Finoguenov et al. 1994).

A search in the WATCH data prior to the discovery revealed that GRS $1915+105$ was active already in 1992 June and July, as was indicated by BATSE (Harmon et al. 1992; see also Paciesas et al. 1994). No WATCH data are available between 1992 April 19 and June 12. An upper limit of 80 mcrab is derived from the April 18 observation. The X-ray light curve shown in Figure 1 in the 6-15 keV energy band represents a period of 169 days (from 1992 April 12 to October 1). The source seemed to vary significantly on timescales from hours to days during the whole observation period (Fig. 2).

A search for pulsations revealed no periodic variations from the source. Our studies set an upper limit of 0.1 Crab (68\% confidence limit) for the pulsed fraction for periods between 15 and $3000 \mathrm{~s}$.

After the discovery, a search for the optical counterpart was initiated at Crimean Astrophysical Observatory, but without finding any object brighter than 18 mag showing variability of more than 0.5 mag inside the 0.5 radius error box given by 


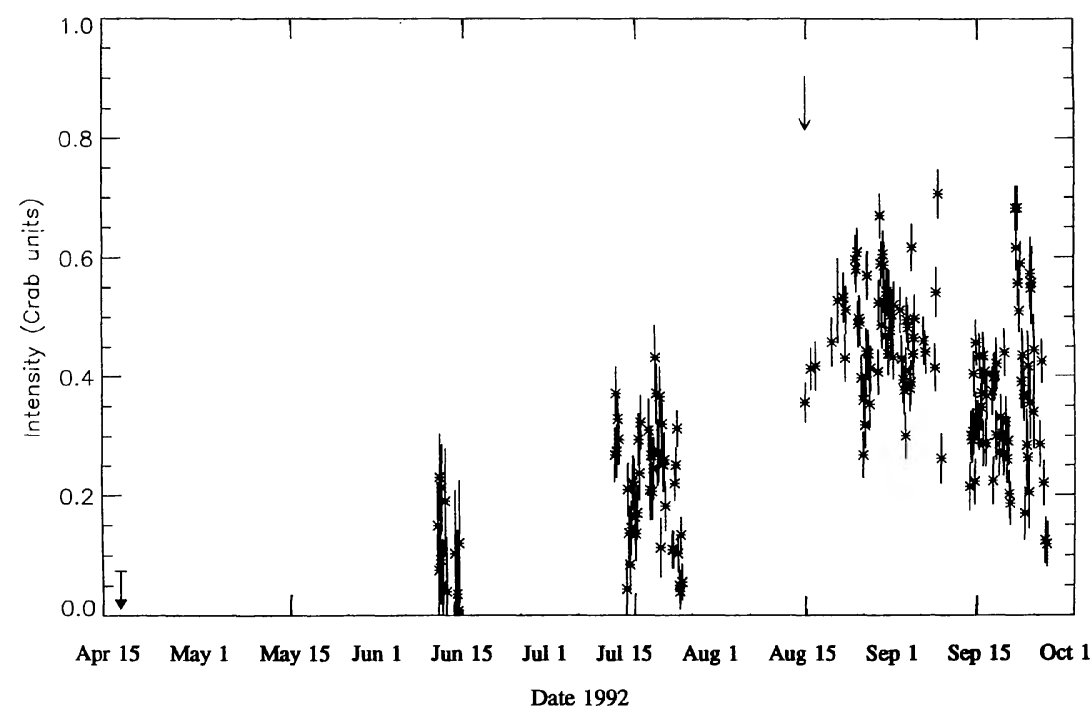

FIG. 1.-Overall X-ray light curve for GRS $1915+105$ in the WATCH 6-15 keV energy band during 1992 April 15-September 30. The arrow indicates the discovery on 1992 August 15 .

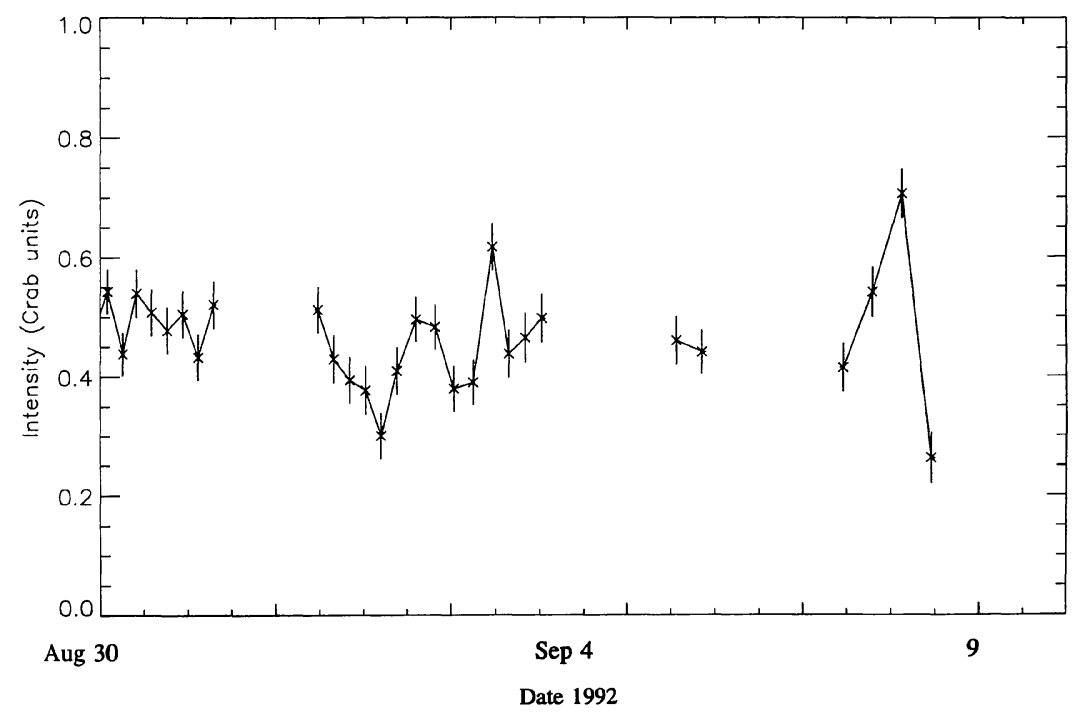

FIG. $2 a$

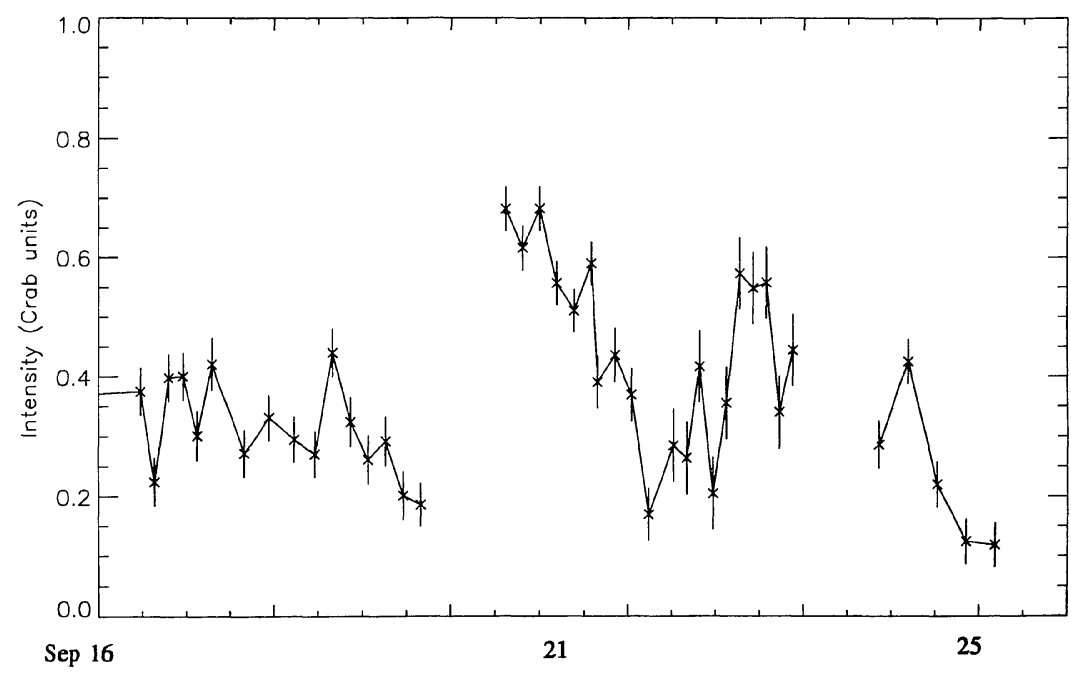

Date 1992

FIG. $2 b$

FIG. 2.-Time variability in GRS 1915+105. (a) Data for 1992 August 30-September 10. (b) Data for 1992 September 16-27. Each point is the integrated flux for $\sim 4$ hours. $\pm 1 \sigma$ error bars are also plotted. 
WATCH. The SIGMA position of GRS $1915+105$ was used when the Schmidt plates taken in 1992 August 18-19 were examined. We derive an upper limit for the magnitude of the optical counterpart of 21 mag.

\section{DISCUSSION}

The X-ray transient GRS $1915+105$ differs strongly from the outbursts of the two Soft X-ray transients detected by WATCH so far, GRS 1124-68 (XN Mus 1991, Brandt et al. 1991 ) and GRO J0422+32 (XN Per 1992, Paciesas et al. 1992; Castro-Tirado et al. 1994). In some ways, it resembles the outburst of the black hole candidate GX 339-4 in 1991 as seen by BATSE (Fishman et al. 1991) and WATCH. Both GRS $1915+105$ and GX 339-4 exhibited an extended early onstate of 1-2 months and slow rising. The spectral fits to GRS $1915+105$ by BATSE (Harmon et al. 1992) are satisfied by a power-law spectrum with a steepening of the spectra from 1992 June to September (evolution of the spectral index from 2.0 to 2.8 ). This is also similar to the GX 339-4 spectral evolution during 1991 August-October (Grebenev et al. 1991). The main difference was, however, the aparition of an ultrasoft component below $3 \mathrm{keV}$ in the GX 339-4 spectrum. At the same time, the flux above $6 \mathrm{keV}$ decreased by a factor of 2 (the transition between the GX 339-4 low and hard states). In the case of GRS 1915+105, although no observations have been reported so far below $6 \mathrm{keV}$, the flux in the $6-15 \mathrm{keV}$ has not decreased as it would be expected if GRS $1915+105$ would have undergone a similar transition. Moreover, the outburst in GX 339-4 lasted for 3 months, whereas in GRS $1915+105$ is still going on. A possible relation of GRS $1915+105$ as the origin of the soft gamma-ray repeater B1900+14 (Mazets et al. 1979) has been discussed ( see Grindlay 1994) due to the observations by BATSE of three very short bursts on 1992 June 19, July 8, and August 19 arising from this region (Kouveliotou et al. 1992), but the radii of the error boxes are of the order of several degrees, and such a relationship is uncertain.

From the search of the optical counterpart of GRS $1915+105$, we derive a limiting magnitude to any optical emitting object of $m_{v}>21$. If we take into account the expected absolute magnitude of the optical counterpart $M_{v} \approx 1.0$, which is the average absolute magnitude for LMXRBs with identified optical companions (van Paradijs 1981). In the direction of GRS $1915+105$ there are several molecular clouds. The first of which is found at a distance of $1.5 \mathrm{kpc}$ (designed as [46, 25] by Myers et al. 1986). Considering the extinction along the line of sight through the first $1.5 \mathrm{kpc}$ as $A_{v}=2.4$ (the hydrogen density is 1 atom per cubic centimeter along the Galactic plane, with a scale height of $50 \mathrm{pc}$ ), it is straightforward to see that the star should have been discovered in our search with $m_{v} \leq 19.8$ if the object is closer than $1.5 \mathrm{kpc}$. For the molecular cloud [46, 25], its mass of $10^{4.5} M_{\odot}$ and size of $0.024 \mathrm{kpc}$ of diameter implies an extinction of a few magnitudes. Therefore we propose a lower limit for the distance of GRS $1915+105$ as $d>1.5 \mathrm{kpc}$. Behind this cloud, the interstellar medium extends over $6.3 \mathrm{kpc}$ before reaching the larger and denser molecular cloud complex [46,59] at $7.8 \mathrm{kpc}$. This complex contains $10^{6.5} M_{\odot}$ in a diameter of $0.148 \mathrm{kpc}$. It also includes several H II regions (Dame et al. 1986). Both complexes are situated in the Sagittarius arm of the Galaxy (Fig. 3).

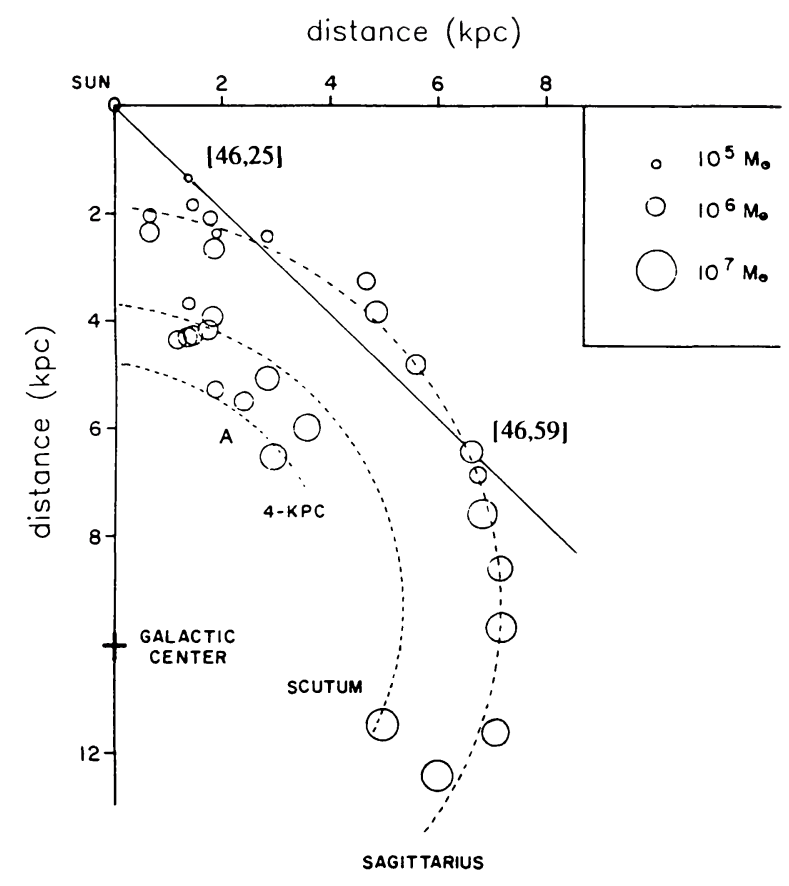

FIG. 3.-Location of the two molecular cloud complexes along the line of sight in the Sagittarius arm of our Galaxy. (Adapted from Dame et al. 1986).

The X-ray flux in the $6-15 \mathrm{keV}$, derived from the WATCH observations, was $3.0 \times 10^{-9}$ ergs $\mathrm{cm}^{-2} \mathrm{~s}^{-1}$ on 1992 August 18-19 (when the Schmidt plates were taken), and the X-ray luminosity $L_{\mathrm{X}}(6-15)=3.6 \times 10^{35}(\mathrm{~d} /(1 \mathrm{kpc}))^{2} \mathrm{ergs} \mathrm{s}^{-1}$, and the ratio $L_{\mathrm{X}}(6-15) / L_{\mathrm{opt}}(V$ band $) \geq 50$ (assuming $M_{v} \approx 1.0$ ). Two likely sites for GRS $1915+105$ could be inside (or nearby) one of the two molecular cloud complexes along the line of sight. If the source is still bright in X-rays, it may be worthwhile to take a deeper CCD frame. If no optical counterpart is found brighter than $m_{v} \sim 25$, infrared observations would be very valuable, specially in the $H$ and $K$ bands, where the extinction is smaller. An infrared detection would allow us to get a spectrum and, together with the infrared magnitude, constrain the distance to the source, and distinguish whether the companion is a LMXB, as we assumed, or a different object, as it happened with Cyg X-3 (Becklin et al. 1972). The massive star from which the compact object in the GRS $1915+105$ binary system originated is more likely to have been born in one of these molecular clouds rather than in the interarm region between the Sagittarius and Scutum arms of our Galaxy. A similar association near the Galactic center between the source 1E1740.7-2942 and a molecular cloud has been recently suggested (Mirabel et al. 1991).

Note added in manuscript.-Recently Mirabel et al. (1993a) have found a variable radiosource inside the SIGMA error box. A variable infrared source has been discovered at the position of the radiosource (Mirabel et al. 1993b; Castro-Tirado et al. 1993), which presumably is the counterpart of GRS $1915+105$.

We are very grateful to the staff of the Evpatoria ground 
station in Ukraine and to those ones of the Lavotchkin and Babakin Space Center for the facilities given in the data acquisition, as well as the hospitality at Crimean Astrophysical Observatory during so many nights spent without identifying any optical counterpart among thousands of stars inside the error box (AJCT). We also thank G. Pizarro, A. Smette, and H.
Zodec for their assistance with the Schmidt plates, and I. Marquez, M. Moles, and J. L. Ortiz for the CCD images taken during their observing time and facilities for data reduction at Instituto de Astrofisica de Andalucia (Granada, Spain). Discussions with H. U. Nørgaard-Nielsen and J. Knude were very fruitful.

\section{REFERENCES}

Becklin, E. E., Kristian, J., Neugebuer, G., \& Wynn-Willians, C. G. 1972, Nature, 239, 130

Brandt, S., Castro-Tirado, A. J., \& Lund, N. 1992, IAU Circ., 5664

Brandt, S., Castro-Tirado, A. J., Lund, N., Lapshov, I., Dremin, V., \& Sunyaev, R. 1991, A\&A, 254, L39

Castro-Tirado, A. J., Brandt, S., \& Lund, N. 1992, IAU Circ., 5590

Castro-Tirado, A. J., et al. 1994, in preparation

Castro-Tirado, A. J., et al. 1993, IAU Circ., 5830

Dame, T. M., Elmegreen, B. G., Cohen, R. S., \& Thaddeus, P. 1986, ApJ, 305,896

Finoguenov, A., et al. 1994, ApJ, 424, 940

Fishman, G. J., Wilson, R., Meegan, C., Harmon, A., \& Brock, M. 1991, IAU Circ., 5327

Grebenev, S., Sunyaev, R. A., Pavlinsky, M., \& Dekhanov, I. 1991, Soviet Astron. Lett., 17(6), 413

Harmon, B. A., Paciesas, W. S., \& Fishman, G. J. 1992, IAU Circ., 5619
Kouvelioutou, C., et al. 1992, IAU Circ., 5567

Lochner, J., \& Whitlock, L. 1992, IAU Circ., 5658

Lund, N. 1985, The WATCH Gamma-Burst Detector for EURECA-I, in X-ray Instrumentation in Astronomy, ed. J. L. Culhane, SPIE 597, 95 Mazets, E. P., et al. 1979, Soviet Astron. Lett., 5(6), 343

Mirabel, I. F., Morris, M., Wink, J., Paul, J., \& Cordier, B. 1991, A\&A, 251, L43

Mirabel, I. F., et al. 1993a, IAU Circ., 5779

- 1993b, IAU Circ., 5830

Myers, P. C., Dame, T. M., Thaddeus, P., Cohen, R. S., Silverberg, R. F., Dwek, E., \& Hauser, M. G. 1986, ApJ, 301, 398

Paciesas, W. S., Briggs, M. S., Harmon, A., \& Wilson, R. B. 1992, IAU Circ., 5580

Paciesas, W. S., et al. 1994, unpublished

van Paradjis, J. 1981, A\&A, 103, 140 